\title{
The Effect of Training in Systemic Thinking Skills on Performance and Perceived Mental Effort When Dealing with Difficult Tasks
}

\author{
Nagdy W Habashy, Howaida M Saber, Ghada A Ahmad* \\ Department of Educational Psychology, Faculty of Education, Minia University, 61519 Minia, Egypt \\ * Correspondence: ghada.abulmagd.a@gmail.com; Tel: +201221098220; Fax: +20 862093320
}

\section{Article information}

Received: 10 May 2020

Revised: 12 June 2020

Accepted: 16 June 2020

\section{Key words}

Systemic Thinking Skills

Performance

Perceived Mental Effort

Difficult and Complex Tasks

Instructional Efficiency

\begin{abstract}
The aim of the current study was to identify the effect of the training program based on systemic thinking skills on the level of performance, and the level of perceived mental effort associated with performance when dealing with difficult and complex tasks. To this aim, a scale of perceived mental effort and a scale of perceived difficulty were prepared, in addition, a program based on the systemic thinking skills which were established by Richmond in 1993 was prepared. The program was applied to a sample of (48) students from university students enrolled in the fourth - year at educational psychology major.

By calculating the significance of the difference between the means of ranks on both performance and perceived mental effort before and after the application of the program using the Wilcoxon test for the related samples, it was found that there is a significant difference between the two means of ranks of performance in favor of the post-measurement, as the level of performance increased significantly after the application of the program. It was also showed that there was a significant difference between the two means of ranks of perceived mental effort obtained on the pre and post-measurements in favor of the premeasurement, as the perceived mental effort level decreased significantly after the application of the program. The instructional efficiency of the intervention based on systemic thinking has been calculated. It had a value of $E=2.76$, which is a positive efficiency, indicating a high level of performance compared with the perceived mental effort after the application of the program. This indicates the instructional efficiency of the proposed training program.
\end{abstract}

\section{Introduction}

Robinson stated that complex dynamic tasks are those tasks that include decision-making problems, or tasks that provide events or changing activities, and require a person to make decisions, make judgments, and reach conclusions and evaluation of events. Robinson also indicated that the difficulty of complex dynamic tasks is determined by the number of elements involved in the task in addition to the number of relationships between those elements [1].

Funke summarized the concept of the problem complexity as being not only limited to the number of variables that must be paid attention to, but also is related to the complex pattern of interconnection between them (linear relationships versus nonlinear relationships), in addition to the ability to control the system and its dynamic manifestations. Besides, he indicated that continuing in the degree of complexity of the problem may also be related to the limited ability to tackle the problem of the person who tries to solve the problem. That training in the method of thinking and processing increases the ability and reduces the complexity of the problem as well as leads to improved performance and reaching satisfactory solutions to the problem [2].

The study of Sengupta \& Abdel-Hamid concluded that developing an understanding of the causal relationships involved in the decision-making task as well as developing the ability to make inference affect both learning and performance level. If the decision maker is unable to understand the causal relationships involved in the decision-making environment, the result will be a low level of performance, but if a person is exposed to interventions that enable him/her to perceive such relationships, the level of performance will improve [3]. This is confirmed by the study of Gigerenzer \& Gaissmaier [4].

One of the approaches that have attracted the attention of many researchers, and which they consider as one of the most appropriate approaches to deal with problems characterized by a complex dynamic nature is the approach based on systemic thinking. Many researchers, [5], [6], [7], [8], [9] summarized the characteristics of systemic thinking and its prominent role in dealing with complex tasks in the following points:

- Holistic thinking: it is a process by which a situation or problem can be understood in a holistic way. It provides a holistic view of the multiple interactions among the component parts of the situation instead of fragmentation into smaller and smaller elements, which impedes understanding and prevents those situations from being solved due to using reduction and analysis.

- Thinking with mutual relationships: through this type of thinking, it is clear that there are more complex causal relationships; in addition to the existence of indirect results and a network of influences for these relationships that can be used to solve more stubborn problems in practical daily life.

- Thinking in models: systemic thinking is a way to build models that help us understand the events and patterns of 
behavior that cause them and reach the basic structure responsible for those events. Hence, systemic thinking includes an analysis of the situation and then re-synthesizing its components flexibly with multiple methods of synthesis and organization in light of what is required to be reached.

- Dynamic thinking: through systemic thinking, individuals realize that there may be unexpected consequences for their actions given that the systems around us are constantly changing; and then they are able to understand the feedback loop, which enables them to modify their actions and behaviors in accordance with the dynamic nature of the problem system and based on their vulnerability to variables exist in the surrounding environment. Therefore, solutions that solve the situation today may become ineffective over time.

- A thinking that considers the results of long-term solutions: Systemic thinking helps to design smart and lasting solutions to problems, encourages thinking about long-term solutions and takes a future view of the evolution of the problem .

Therefore, many of recommendations emerged suggest that students should be taught systemic thinking through training programs separate from the school curriculum or through the curriculum provided to them, and designing these training programs in a systemic way. This is due to what was found in the studies of Sterman; Paich \& Sterman; Diehl \& Sterman that the level of individuals was below average in terms of the systemic aspect when dealing with simple systems, and that performance was noticeably reduced when the easiest levels of dynamic complexity is introduced, and learning was weak and slow even with repeating the attempts and providing sufficient time to perform.

Those studies attributed such poor performance to individuals' adherence to linear thinking with limited relationships, which weakened their ability to correct reasoning and to show the multiple factors that influence the problem behavior system. In addition, individuals failed to determine the circular causal relationships between those factors, did not pay attention to the temporal effects on the development of the problem behavior, and were concerned with treating the symptoms of the problem instead of the real causes, as they take direct solutions without paying attention to the long-term results of those solutions. They showed also less understanding of the structure of the problem, as well as their analyzes were characterized by superficiality [10], [11], [12], [13].

In recognition of this gap, a set of experimental studies investigated the effectiveness of systemic thinking interventions in improving performance on complex dynamic problems. The study of Manni \& Maharaj found that systemic thinking skills are linked to performance on complex tasks, and the study indicated that the performance pattern of individuals who achieved a high level of performance differed significantly from the performance pattern of individuals with low performance, as individuals who achieved a high level of performance show a better understanding of the system structure before beginning to develop the treatment and decision-making strategies [14].

It was also found in the study of Abdyrov et al. that students have demonstrated more efficient levels in the cognitive processing of learning tasks and in the theoretical and practical activities assigned to them, after subjecting them to a training program to develop their systemic thinking skills. The study indicated that the interventions based on systemic thinking contributed to improving the effectiveness and efficiency of learning, and also contributed to the development of cognitive processes and activities, to the smart performance of learners as well as to the development of their capabilities to carry out critical analyzes that are necessary to develop knowledge associated with a particular field [15]. In addition, Viacheslave also supported the interventions based on systemic thinking and their impact on raising performance on complex problems [16]. At the national level, Hassan explored the effectiveness of a program in developing the systemic thinking skills among dogmatic college students and its effect on solving complex educational problems. It was revealed in this study that the proposed program is effective in improving the level of performance on complex educational problems among that category [17].

From the above-mentioned, it is found that research in the field of systemic thinking in the local and Arab environment is relatively recent, especially when dealing with systemic thinking as an approach to solving life problems that are characterized by the complex dynamic nature. This motivated the researcher to design a proposed program in order to develop systemic thinking skills among university students and identify its effect at performance level when dealing with complex dynamic problems.

Furthermore, several studies aimed at determining the level of perceived mental effort associated with performance on tasks with varying difficulty levels. The study of Renkl \& Atkinson found that students showed a high level of perceived mental effort during their engagement in performance on tasks of relative difficulty. To interpret that, each of them explains that most students do not attempt to use their cognitive abilities effectively as well as do not engage in processes of selfinterpretation of problems, especially when they encounter problems with which they have no previous experience; they make random attempts and general procedures that negatively affect the level of performance and cause a high level of cognitive load, compared with the expert person who makes less mental effort when dealing with those given tasks due to their easiness for him/her "[18].

Kalyuga et al. added that "the expert person has the cognitive schemata that he/ she uses when dealing with difficult tasks that allow him to reduce the load imposed on the working memory in a meaningful way. As when the expert person confronts a problem, s/he is, in this case, able to distinguish the relationships among the multiple elements of the problem, and $\mathrm{s} /$ he also processes them as a single unit. Although these units have a higher level or multiple relationships, they require less treatment compared with multiple units from lower levels. The more of these diagrams (schemata) flow, the more experienced the learner is and the less significant mental effort the learner makes" [19].

Accordingly, a large number of studies, such as Ayres; Sweller; Zheng \& Cook recommended the importance of training students in cognitive processing of information skills that contribute to the formation of cognitive schemes to improve the level of performance and to meet task requirements with the lowest level of mental effort [20], [21], [22].

A great deal of literature and theoretical frameworks emphasized the effectiveness of systemic thinking interventions in improving performance and reducing the perceived mental effort associated with it. Doyle indicated that systemic thinking works to organize information and enter it into long-term memory in an integrated, organized and logical manner, which 
allows for the formation of cognitive schemes and recalling them in problem-solving situations with speed and accuracy, which improve the level of performance and reduce the mental effort associated with it [23].

Clark et al. stated that the perspective which is based on systemic thinking provides a more coherent organization of knowledge, and that this organization is necessary to enable individuals to make optimal use of the working memory sources and to reduce restrictions on the working memory capacity which reduces their cognitive load [24]. Pass et al. hypothesized that the individuals who learn in a systemic way must perform better than those who learn in the traditional way, and that the systemic approach may contribute to the reduction of mental effort when dealing with complex tasks [25].

Based on the previous hypotheses, Brewster studied the effectiveness of the systemic approach in comparison with the reductive approach in the ability to use working memory sources and the ability to have cognitive processing of complex tasks dynamically. The participants who relied on systemic thinking showed more accurate and coherent mental models, as well as greater credibility in performance due to the clarity and coherence of their mental models. They also demonstrated a more effective use of the working memory, compared with the participants who depended on the reductive method, which led to a decrease in their mental effort as measured by a graded scale of 1: 9 to represent the levels of mental effort when dealing with complex tasks [26]. In contrast, Best drew a comparison between the effect of the systemic approach and the traditional method of teaching on performance and the associated mental effort or on building cognitive schemes for beginners from university students. It was found that there is no significant difference between the systemic and traditional methods on performance and the associated mental effort nor on building and forming cognitive schemes [27].

It is clear from the above-mentioned that the results of the two studies conflict with regard to the effect of systemic thinking on the performance and the perceived mental effort among students, in addition to the lack of experimental studies conducted in this field, which called for studying the effect of a program to develop systemic thinking skills on the level of perceived mental effort when performing difficult and complex tasks among university students. In addition, in this study, the instructional efficiency of the intervention based on systemic thinking as an approach to integrate between the level of perceived mental effort and the level of performance on difficult tasks was calculated, because there is no experimental nor empirical evidence for such efficiency.

Pass and Van-Merienboer pointed out that measuring mental effort is a good addition to performance measurements, as combining perceived mental effort scores and performance can produce more sensitive results to the cognitive effort of training or the test environment compared with the results of the measurements of the effort only or performance only. Each of them also recommended the necessity for using this approach in future research in order to determine the efficiency of the training programs and educational interventions. They provided a mathematical input to the efficiency of educational interventions from the relationship $(\boldsymbol{E}=(\boldsymbol{P}-\boldsymbol{R}) / \sqrt{\mathbf{2}})$, where $\boldsymbol{P}$ indicates the standard score for performance, and $\boldsymbol{R}$ indicates the standard score for perceived mental effort by learners and associated with performance on the post-test [28]. De Jong explained this relationship as follows:
- If P-R>0, this indicates positive instructional efficiency: i.e. a high level of performance combined with a low level of mental effort.

- If $\mathrm{P}-\mathrm{R}<0$, this indicates negative instructional efficiency: i.e. a low level of performance combined with a high level of mental effort.

- When both mental effort and performance are equal, meaning that they are both low in level or high in level, this indicates that the experimental situations are ambiguous and that their effectiveness is not predictable [29].

In the current study, the researcher relied on the systemic thinking skills which were established by Richmond in 1993 , because they are among the skills that have been mostly used in order to develop systemic thinking among individuals, especially when dealing with complex dynamic problems. These skills are represented in the following seven skills:

\section{- Dynamic Thinking Skill}

Dynamic thinking is the ability to observe gradual changes which are taking place in the behavior of variables that extend over time; this means that the individual needs to place the current situation in the context of a time gradient (scale) by drawing a course of behavior; so that this course has temporal divisions represented in the current state in addition to one or more than future courses [30]. The dynamic thinking skill is employed in case of exposure to the situation of taking one of the two options available based on the future results of these options. In this case, each choice as well as its results must be studied separately in the short and long term, taking into account the impact of time changes on the results of the choice that will be taken so that the problem does not get worse [31].

\section{- System-as-Cause Thinking}

In this skill, the individual must think about the dimensions and elements of the system that constitute his behavior, and realize that this observed behavior can be the result of the relationships that the laws of nature imposed on these elements instead of the decisions and actions of a particular person [30]. According to this skill, when a person encounters a problem, s/he begins to identify the system that creates the behavior of the problem, and then looks for the factors that make up the system's structure that caused the problem [31].

\section{- Operational thinking skill}

A practitioner of operational thinking can distinguish the causal relationships between events and think about how things and phenomena occur instead of thinking about them in abstract form which is greatly far from reality [30]. In this case, the reasons that led to specific results are considered, in addition to an explanation of the processes that take place in the problem system. Operational thinking can be employed when it is necessary to comment on the causes of a problem, identify what is wrong in the conclusion, and state the reasons that seem more logical [31].

\section{- Closed-loop thinking skill}

When individuals think of using closed-loops, they see the world as a set of continuous processes that depend on one another, rather than seeing them as a list of one-dimensional relationships [30]. The stories of causal closed-loops are 
expressed using variables and arrows (links) that show how one of the variables affects the other to make the situation better or worse. The effect here usually provides feedback that influences one or more of the causes; rather, the causes themselves affect each other [32]. The closed-loop thinker must understand that every decision or action that is taken will have unintended consequences that are responsible for shaping the situation upon which decisions will be made in the future. According to this skill, a systemic thinker has to comment on the source of the deterioration of the problem system situation [31].

\section{- Forest thinking skill (holistic thinking)}

This skill enables individuals to see the system of relationships and events in a holistic way rather than focusing on the elements in an isolated way; it means going beyond the analysis to the structure [30]. According to this skill, all or some elements of the system are improving; while, the behavior of the system does not show any improvement; it may even show a breakdown. An explanation is required as to why this is happening. Forest thinking skill can be applied by emphasizing that the whole is greater than the sum of its parts, and that what achieves the goals and benefits of the system is harmony and consistency between the components [31].

\section{- Quantitative Thinking Skill}

In quantitative thinking, the thinker must understand that every decision made or every behavior that is taken is the result of a set of attitudes that the individual is biased towards, or is the result of a set of motives that are responsible for shaping the status quo. The person in charge of this skill must determine the motives and attitudes that prompted the owners of the problem to make certain decisions, in addition to identifying the stakeholders for whom the system seeks to achieve their desires and goals [30].

\section{- Scientific thinking skill}

In scientific thinking, a person must realize that $\mathrm{s} / \mathrm{he}$ is thinking of models based on hypotheses, and that these hypotheses are always limited in application. In addition, these hypotheses must be tested and reviewed periodically in order to improve the dynamic nature of the systems around us; which constantly changes itself so as to adapt to the conditions and challenges of reality around us. The person in charge of this skill should follow-up the application of solutions to problems and evaluate their results in the long run in light of the gains and losses resulting from the application of these solutions [30].

Based on the above-mentioned, the problem of the current study can be summarized in the following questions:

1. Is there a statistically significant difference between students' level of performance on complex tasks before and after applying the training program?

2. Is there a statistically significant difference between students' level of perceived mental effort when dealing with complex tasks before and after applying the training program?

3. What is the instructional efficiency of the training program in light of students' level of performance and their level of perceived mental effort?

\subsection{Hypotheses}

1) There is a statistically significant difference between the mean ranks of students' performance on complex tasks before and after applying the training program in favor of post-measurement.

2) There is a statistically significant difference between the mean ranks of students' perceived mental effort when performing complex tasks before and after applying the training program in favor of pre-measurement.

3) The training program will show a positive instructional efficiency among students after post-measurement.

\section{Materials}

\subsection{Training program}

The training program, which is based on systemic thinking skills described by Richmond in 1993 and revised in 1997, was prepared in accordance with the operational definition of each skill. The program included 14 training sessions: the first session contained a theoretical background in order to familiarize students with the concept of systems and their properties and how to apply those characteristics to one of the realistic systems around us (e.g. the educational system). The activities assigned to students were performed in a collaborative way. The second session aimed at introducing students to the concept of systemic thinking and distinguishing it from linear thinking, in addition to a presentation of its characteristics, as well as an explanation of the importance of gaining the ability to use it for individuals.

As for the sessions from the third to the twelfth, they included activities and exercises aimed to train students in using the seven skills of systemic thinking in a separate and independent way, through realistic situations and scenarios which the individual may witness or be exposed to in his/her professional and personal life. In the thirteenth and fourteenth sessions, tasks and scenarios were presented, in which the trainees were asked to reach certain conclusions using the combined systemic thinking skills.

The training program included the general aim of each session and the operational objectives derived from it, as well as the strategies of brainstorming, cooperative learning and modeling were identified as teaching techniques. It also contained audiovisual educational presentations to present certain theoretical concepts, plans and structural models of problems and scenarios presented in the program. The program has been validated by submitted it to experts whose suggestions and modifications have been taken into consideration. After experts' review, they agreed on the possibility of its implementation.

\subsection{Perceived mental effort questionnaire}

The perceived mental effort questionnaire was prepared in accordance with the perspective of the theory of Cognitive Load and in light of many measurement scales that have been mentioned in previous studies. The scale, in its preliminary 
form, consisted of 14 items which respond to through a 5-point Likert rating by choosing one of the following alternatives (very high degree, high degree, medium degree, low degree, very low degree).

After submitting the scale to specialized jury members as well as subjecting it to confirmatory factor analysis of the first degree, six items were retained, namely: -I made a mental effort to understand the content of the topic, - I resolved to answer questions despite their difficulty, - I have read the text more than once in order to understand it, - I made a mental effort to distinguish between the information in the topic, - I thought and meditated to solve the questions correctly, - performing the task requires an appropriate mental effort. The standard regression weights for these items of the scale ranged between 0.545 and 0 . 839 , which are acceptable values as well as significant at the level of 0.001. In addition, all good fit indices came in the ideal range as shown in the following table:

Table 1: Good Fit Indices of Perceived Mental Effort Questionnaire, $\mathrm{N}=190$

\begin{tabular}{|c|c|c|c|c|c|c|c|c|c|}
\hline $\bar{\partial}$ & $\underset{z}{z}$ & $\Xi$ & 堊 & 尩 & $\overrightarrow{\mathbf{Z}}$ & $\widetilde{\Omega}$ & $\Omega$ & 园 & $\underset{b}{\mathbb{2}}$ \\
\hline
\end{tabular}

$\begin{array}{lllllllll}1.393 & 0.983 & 0.966 & 0.944 & 0.581 & 0.594 & 0.990 & 0.990 & 0.045\end{array}$

Hence, the model has a good fit of the data being tested. In addition, the internal consistency reliability of the scale structure was calculated, and its coefficient of alpha-Cronbach was 0.783 , which is a high value for reliability.

\subsection{Task perceived difficulty questionnaire}

After reviewing many measurement questionnaires that have been included in previous studies, the task perceptive difficulty questionnaire was developed in accordance with the perspective of cognitive load theory. In its preliminary form, the questionnaire consisted of 7 items to be answered through a 5point Likert rating by choosing one of the following alternatives (very high degree, high degree, medium degree, low degree, very low degree).

After submitting the scale to specialized jury members as well as subjecting it to confirmatory factor analysis of the first degree, five items were retained, namely: -topic content is difficult to understand, - topic contains complex and mutually dependent information, - topic contains multiple information that is difficult to be read easily, - I had difficulty understanding the topic content due to a lack of my information, - I could not determine the best solution because of the complex relationships among the subject elements. The standard regression weights for these items of the scale ranged between 0.462 and 0.766 , which are acceptable values as well as significant at the level of 0,001 . Besides, all indices of good fit came in the ideal range as shown in the following table:
Table 2: Good Fit Indices of Task Perceived Difficulty Questionnaire, $\mathrm{N}=193$

\begin{tabular}{|c|c|c|c|c|c|c|c|c|c|}
\hline $\bar{\Xi}$ & $\underset{3}{3}$ & $\Xi$ & Z & 元 & 忞 & ح & $\Omega$ & 鳬 & $\underset{2}{2}$ \\
\hline 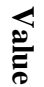 & 1.586 & 0.877 & 0.743 & 0.725 & 0.694 & 0.826 & 0.885 & 0.887 & 0.055 \\
\hline
\end{tabular}

Hence, the model has a good fit of the data being tested. Besides, the internal consistency reliability of the scale structure was calculated, and its coefficient of alpha-Cronbach was 0.760 , which is a high value for reliability.

\subsection{Task}

Two tasks characterized by complexity and relative difficulty were used to perform, as the participants of the experimental group were assigned to perform one of them before the application of the training program, which was entitled "Cattle Breeding in Hailu Tribe" and the other task was assigned to the participants of the experimental group to perform after the manipulation of the training program, which was entitled " Māori Fishing". Two different tasks have been applied to avoid the influence of the experience factor on the level of performance as well as the level of perceived mental effort, and to make sure that all effect on the dependent variables is due to the training program under study.

Sedney pointed out that these two tasks are equivalent in terms of structure and level of difficulty [33]. To make sure of that, the perceived difficulty questionnaire was administered to 57 participants after being assigned to perform each of the two tasks. Then, the correlation between the ranks of the scores of the perceived difficulty on both tasks was calculated. The correlation coefficient reached 0.787 , which is a high value and significant at the level of 0,001 . This is an evidence of the equivalence of the two tasks in terms of the level of perceived difficulty.

With regard to determining the difficulty level for each of the two tasks, the significance of the difference between the mean of the perceived difficulty on each of the two tasks and the hypothetical mean was calculated, which is estimated at the value of 15 as shown in the following table:

Table 3: T-test results of the significance of difference between the mean of perceived difficulty on both tasks and the hypothetical mean(=15)

\begin{tabular}{|c|c|c|c|c|c|c|}
\hline Indicators & $\begin{array}{l}3 \\
\stackrel{3}{8}\end{array}$ & 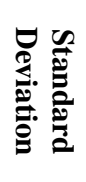 & 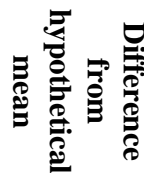 & 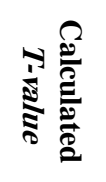 & 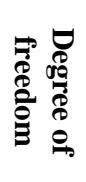 & 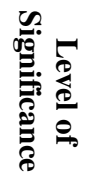 \\
\hline $\begin{array}{c}\text { Perceived } \\
\text { difficulty of } \\
\text { Māori Task }\end{array}$ & 21.82 & 2.331 & 6.825 & 22.105 & 56 & 0.001 \\
\hline $\begin{array}{c}\text { Perceived } \\
\text { difficulty of } \\
\text { Helieu Task }\end{array}$ & 21.68 & 1.692 & 6.684 & 29.826 & 56 & 0.001 \\
\hline
\end{tabular}


According to what is shown in (Table 3), the difference between the mean of perceived difficulty on both tasks and the hypothetical mean $(=15)$ is a significant and in favor of the perceived difficulty of the two tasks, which indicates the high level of difficulty of the two tasks. In addition, the difficulty coefficient for each item included in each task was calculated, and the values of those parameters were as shown in the following table:

Table 4: Difficulty coefficients for each question in each task and their means

\begin{tabular}{|c|c|c|c|c|c|c|c|c|c|}
\hline 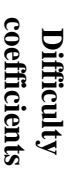 & 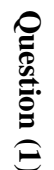 & 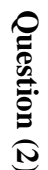 & 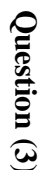 & 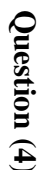 & 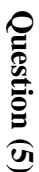 & 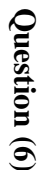 & 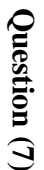 & 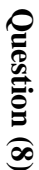 & 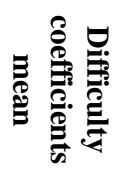 \\
\hline
\end{tabular}

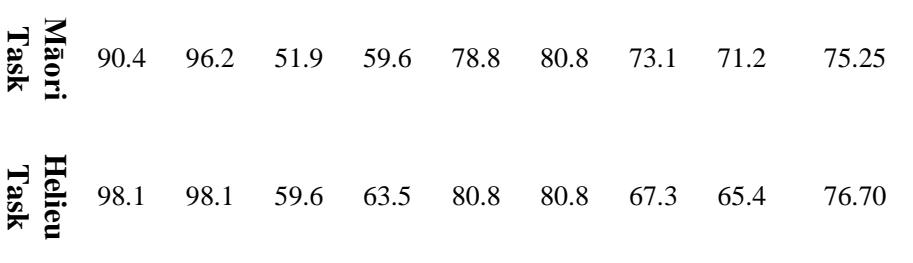

It is clear that the difficulty coefficients for each question are high, which indicates the high level of the general difficulty of the task, as the mean of difficulty coefficients for the Māori task are 75.25 , and the mean of difficulty coefficients for the Helieu task are 76.70 .

\section{Method}

The main sample of application consisted of (48) male and female students enrolled in the Faculty of Education, fourthyear, Major of Educational Psychology, who have a literary background. The participants were assigned to perform the task of the Helieu tribe, then a questionnaire of the perceived mental effort was administered to them after they finished performing the task in order to conduct the pre-measurements of both performance and the perceived mental effort.

The training program for developing systemic thinking skills has been applied to the participants. The implementation of the program took five weeks, with three sessions per week. Where the application started on 15/ 11/ 2019 and ended on 21/ 12/ 2019. After completing the application of the training program, the participants were assigned to perform Māori task, then a questionnaire of the perceived mental effort was administered to them after finishing performing the task in order to conduct the post-measurements of both performance and the perceived mental effort. Data of the pre and post-measurements were subjected to statistical analyzes in order to obtain the results of the study.

\section{Findings and discussion}

\subsection{Findings and discussion of the first hypothesis}

In order to find out the effect of the training program on the level of performance on difficult and complex tasks, the significance of the difference between the mean ranks of performance on the two tasks was calculated before and after the implementation of the program, using the Wilcoxon test for the related samples. The following table shows these results:

Table 5: Wilcoxon test results of the significance of difference between the mean ranks of performance before and after program implementation, $\mathrm{N}=48$

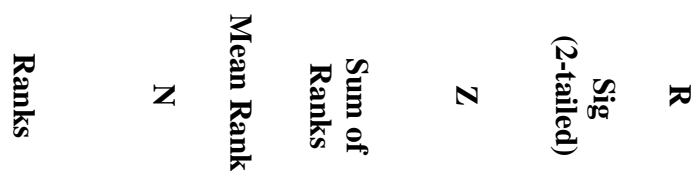

\begin{tabular}{|c|c|c|c|c|c|c|c|}
\hline \multirow{2}{*}{ 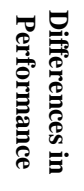 } & Negative Ranks & $0^{\mathrm{a}}$ & .00 & .00 & \multirow{2}{*}{$\begin{array}{c}-6.088^{\mathrm{a}} \\
\text { Based } \\
\text { on } \\
\text { negative } \\
\text { ranks }\end{array}$} & \multirow{2}{*}{.000} & \multirow{2}{*}{0,62} \\
\hline & $\begin{array}{l}\text { Positive Ranks } \\
\text { Ties }\end{array}$ & $\begin{array}{c}48^{\mathrm{b}} \\
0^{\mathrm{c}}\end{array}$ & 24.50 & 1176.00 & & & \\
\hline
\end{tabular}

a. indicates that the level of performance before the application of the program is higher than the level of performance after the application of the program.

b. indicates that the level of performance after the application of the program is higher than the level of performance before the application of the program.

c. indicates that the performance level before the application of the program equals the level of performance after the application of the program.

As shown in (Table 5), the number of the participants who have higher scores (levels) of performance after the implementation of the program is (48), and they are all members of the main sample and the mean of ranks of them is (24.50); whereas, the number of participants who have lower scores level of performance after the application of the program is zero and the mean of ranks of them is zero. Hence, it is clear that there is a statistically significant difference at the level of 0.001 between the man ranks of performance on the task before and after applying the program in favor of post-measurement, as the value of $\mathrm{Z}$ is $(-6.088 \mathrm{a})$, depending on the negative ranks, as well as the effect size $(R)$ is 0.62 , which is a high effect size. This points out the strong effect of the training program (independent variable) on the improvement of the level of performance on the task.

Participants' acquisition of systemic thinking skills made them more able to process information and more aware of the interrelationships among variables. Moreover, those skills contributed to the improvement of their thinking regarding causal relationships, and raised their ability to infer and understand the basic structures behind the problems which led to an improvement in the level of performance on those problems. This is consistent with the Gary \& Wood study which suggested that developing the causal inference ability can reduce task requirements to levels that can be managed, and that the reinforcement of hierarchical thinking or network thinking based on identifying different mutual relationships between the variables can lead to the formation of a more accurate and complete mental model for the task, and this mental model enables the individual to reach many conclusions, that lead to an improvement in the level of performance on difficult and complex tasks [34]. 
Besides, the activities that the participants were exposed to through the training program also contributed to increasing their ability to understand the dynamic nature of the problems, which contributed to the improvement of the level of performance on the tasks, and this was represented in choosing the most appropriate procedures that lead to solving problems in the long term. This finding is in consistency with the Richmond study, which showed that students' acquisition of systemic thinking skills develops the ability to make decisions, and also helps them understand complex problems that are dependent on each other, by forming accurate and complete mental representations of these problems, which enables them to make better decisions regarding the possible interventions for these problems and to be able to solve them permanently rather than overcoming their symptoms [35].

Findings of the current study are consistent with the study of Manni \& Maharaj, which concluded that systemic thinking skills are linked to performance on complex tasks [14], as well as the study of Abdyrov et al. which revealed that students showed more effective levels in the theoretical and practical activities assigned to them after subjecting them to a training program for developing the skills of systemic thinking [15]. In addition, the findings are also in agreement with the findings of the studies of Hassan and Viacheslave, which both showed the effectiveness of interventions based on systemic thinking in raising the level of performance on complex problems [17], [16]. While, the findings of this study are not consistent with Adam's study, that revealed that there is no significant improvement in students 'performance on complex problems, due to the fact that systemic thinking interventions in that study were only weekly theoretical lectures without any practical exercises or activities that had a weak impact on the formation of mental models that lead and direct behavior [36].

\subsection{Findings and discussion of the second hypothesis}

In order to find out the effect of the training program on the level of perceived mental effort when performing difficult and complex tasks, the significance of the difference between the mean ranks of perceived mental effort when performing the two tasks was calculated before and after the implementation of the program, using the Wilcoxon test for the related samples. The following table shows these results:

Table 6: Wilcoxon test results of the significance of difference between the mean ranks of perceived mental effort before and after program implementation, $\mathrm{N}=48$

\begin{tabular}{|c|c|c|c|c|c|}
\hline 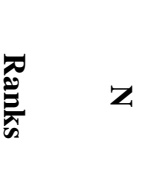 & 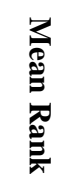 & 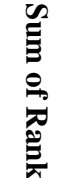 & $\mathbf{N}$ & 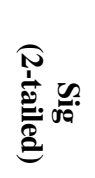 & $\vec{N}$ \\
\hline
\end{tabular}

\begin{tabular}{|c|c|c|c|c|c|c|c|}
\hline \multirow{2}{*}{ 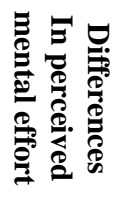 } & $\begin{array}{l}\text { Negative } \\
\text { Ranks }\end{array}$ & $45^{\mathrm{a}}$ & 25.00 & 1125.00 & \multirow{3}{*}{$\begin{array}{c}-5.944^{\mathrm{a}} \\
\text { Based } \\
\text { on } \\
\text { negative } \\
\text { ranks }\end{array}$} & \multirow{3}{*}{.000} & \multirow{2}{*}{0,61} \\
\hline & $\begin{array}{l}\text { Positive } \\
\text { Ranks }\end{array}$ & $2^{\mathrm{b}}$ & 1.50 & 3.00 & & & \\
\hline & Ties & $1^{\mathrm{c}}$ & & & & & \\
\hline
\end{tabular}

a. indicates that the level of perceived mental effort after the application of the program is less than the level of perceived mental effort before the application of the program.

b. indicates that the level of perceived mental effort after the application of the program is higher than the level of perceived mental effort before the application of the program.

c. indicates that the level of mental effort perceived before the application of the program equals the level of perceived mental effort after application of the program.

According to what is shown in (Table 6), the number of participants who have lower scores on the perceived mental effort after the application of the program is (45), and their mean of rank is (25); while, the number of participants who have higher scores on the perceived mental effort after applying the program is (2) and their mean of rank is (1.50). Furthermore, one participant is found to have equal perceived mental effort before and after applying the program. Hence, it is clear there is a statistically significant difference at the level of 0.001 between the mean ranks of perceived mental effort when performing the task before and after the implementation of the program in favor of pre-measurement, as the value of $\mathrm{Z}$ is (-5.944a), depending on the negative ranks, and the effect size $(\mathrm{R})$ reached 0.61 which is a high effect size, indicating the significant impact of the program (the independent variable) on the reduction of the perceived mental effort when performing complex and difficult tasks.

Training the study participants in systemic thinking skills leads to an increase in their ability to analyze attitudes and issues into the largest possible number of variables, in addition to increasing their ability to make a link among these variables according to the mutual and cross-cutting relationships among them in order to form an accurate and complete mental model of the phenomenon that enables them to reach many conclusions. This model contains a large amount of information and is treated as a single unit in the working memory, which contributes to reducing the load on the working memory and provides additional capacity in it to carry out more cognitive processing without making more mental effort. Clark et al. is in agreement with this finding, as they pointed out that the perspective that relies on systemic thinking provides a more coherent system of knowledge and that this system is necessary to enable individuals to make optimal use of working memory sources and reduce restrictions on working memory capacity which reduces the cognitive load which they have [24].

In addition, the participants' habitual use of systemic thinking skills, spending enough time in addressing many situations, and creating cognitive plans (schemata) for these situations using these processes make their performance automatic when exposed to similar situations. In this case, the requirements imposed on working memory will be within the limits of the allocated capacity, which reduces the perceived mental effort when dealing with such situations. This is confirmed by Doyle who pointed out that systemic thinking works to organize information and enter it into the long-term memory in an integrated, organized and logical manner, which allows the formation of cognitive schemata and calling them back in 
problem-solving situations with speed and accuracy, which improves the level of performance and reduces the mental effort associated with it [23].

Moreover, training the participants in systemic thinking skills develops their inference ability that makes them more able to respond to situations and tasks with a high level of complexity, which reduces the mental effort when dealing with such environments. That finding is supported by Gary \& Wood who argued that the development of inference ability can reduce task requirements to manageable levels. Hence, the cognitive load associated with the task is within the limits of the capacity of the working memory [34].

Findings of the current study are also consistent with the study of Brewster in which participants who were dependent on systemic thinking showed more accurate and coherent mental models, as well as greater credibility in performance due to the clarity and coherence of their mental models. In addition, they demonstrated also more efficient use of working memory, compared with those participants who were dependent on the reductionist method. This led to a decrease in their mental effort, as indicated by a scale of 1: 9 to represent levels of mental effort when dealing with complex tasks [26]. Whereas, the findings of this study are not in consistency with Best's study, which showed that there is no significant difference between the systemic and traditional methods in both performance and the associated mental effort or in forming cognitive schemata [27].

\subsection{Findings and discussion of the third hypothesis}

In order to calculate the instructional efficiency of the training program, the mean standard scores of the performance scores on the task were calculated, as well as the mean standard scores for the scores of perceived mental effort related to the performance on the task were calculated after the completion of the application of the program. The mean standard scores of performance was $(\boldsymbol{P}=\mathbf{3 . 0 4 5})$ and the mean standard scores of perceived mental effort was $(\boldsymbol{R}=\mathbf{- 0 . 8 6})$. By applying the instructional efficiency equation $\mathbf{E}=(\mathbf{P}-\mathbf{R}) / \sqrt{\mathbf{2}}$, it was found that the value of instructional efficiency is $\mathbf{E}=\mathbf{2 , 7 6}$ which is a positive value and this indicates a high level of performance compared with the perceived mental effort after applying the program which indicates the instructional efficiency of the training program under investigation.

\section{Conclusion}

Systemic thinking is considered as one of the most appropriate and effective interventions, especially when dealing with difficult and complex situations and problems. Systemic thinking enables individuals to form a mental plan or model (schema) for the phenomenon under study, and this schema contain many elements interconnected with each other with a network of relationships, enabling individuals to reach many conclusions, solutions and multiple decisions depending on the requirements for the situation or the dynamic nature of the phenomenon, which contributes to improving the level of performance on such situations, as well as reduces the perceived mental effort when dealing with these tasks, because the schema which is representative of the phenomenon will be dealt with as one unit, which reduces the load on the working memory and provides additional capacity in the working memory to do more processing. With continuous and repeated use of systemic thinking skills when exposed to many different situations, the performance of individuals will shift to be mechanical as well as automatic, and will be characterized by accuracy and speed.

Therefore, it is recommended that students should be taught and trained in the skills of systemic thinking in order to use those skills continuously and repeatedly for raising the efficiency of individuals when dealing with difficult and complex problems, which is a necessary and important requirement in the era of cognitive complexity which is full of rapid changes in political, economic, educational, social, professional and personal areas.

\section{References}

[1] Robinson P. Task complexity , task difficulty, and task production: Exploring interactions in a componential framework. Applied Linguistics. 2001 22(1): 27-57

[2] Funke J. Solving complex problems: Exploration and control of complex systems. In: Sternberg RJ , Frensch PA (eds). Complex problem solving Principles and mechanisms. Lawrence Erlbaum Association ,NJ: Hillsdale; 1991; pp.185-222.

[3] Sengupta K, Abdel-Hamid TK. Alternative conceptions of feedback in dynamic decision environments: An experimental investigation. Management Science. 1993 ; 39(4): 411- 428 .

[4] Gigerenzer G, Gaissmaier W. Heuristic decision making. Annual Review of Psychology . 2011; $62: 451-482$.

[5] Senge PM. The fifth discipline: The art and practice of the learning organization. $1^{\text {st }}$ ed. New York : Doubleday/ Currency; 1990.

[6] Goodman M, Karash R. Six steps to thinking systemically. The Systems Thinker. 1995; 6(2): 16-18.

[7] Arnson D. Introduction to systems thinking variable . 1996. Available from: http://www.thinking.net/ systems_thinking/intro_to_st.html.

[8] Richmond B. The thinking in systems thinking: How can we make it easier to master. The Systems Thinker. 1997a; 8(2): 1-5.

[9] Bartlett G. Systemic thinking: A simple thinking technique for gaining systemic focus. The International Conference on thinking. 2001. Available from: http://www.Scribd.com/doc/11532348/systemic _ thinking

[10] Sterman JD. Misperceptions of feedback in dynamic decision making. Orgnizational Behaviour and Human Decision Processes. 1989a; 43(3): 301 335 .

[11] Sterman JD. Modeling Managerial Behavior: Misperceptions of feedback in a dynamic decision experiment. Management Science. 1989b; 35(3): 321 339.

[12] Paiche M, Sterman JD. Boom, Bust, and failures to learn in experimental markets. Management Science. 1993; 39(12): 1439-1458.

[13] Dielh E, Sterman JD. Effects of feedback complexity on dynamic decision making. Organizational Behavior and Human Decision Processes. 1995; 62(2): 198- 215.

[14] Maani KE, Maharaj V. Links between systems thinking and complex decision making. System Dynamics Review. 2004; 20(1): 20- 48.

[15] Abdyrov A, Galiyev T, Yessekeshova U, Aldabergenova S, Alshynbayeva $\mathrm{Z}$. On systems thinking and ways of building it in learning. International Journal of Environmental and Science Education. 2016; 11(18): 11149- 11161.

[16] Viacheslave M. Systems thinking and collective problem solving practices. Conference Paper,November 2018. Available from:

http://www.researchgate.net

[17] Hassan DA. The effect of developing systemic thinking skills on complex educational problem solving for the dogmatic university students. A Dissertation submitted in partial fulfillment of the requirements of the degree of doctor of philosophy in educational psychology, University of Tanta, Egypt. 2010 . 
[18] Renkl A, Atkinson R. Structuring the transition from example study to problem solving in cognitive skill acquisition: A cognitive load perspective. Educational Psychologist. 2003; 38: 15-22.

[19] Kalyuga S, Ayres P, Chandler P, Sweller J. The expertise reversal effect. Educational Psychologist. 2003; 38: 23- 31.

[20] Ayres P. Empact of reducing intrinsic cognitive load on learning in a mathematical domain. Applied Cognitive Psychology. 2006; 20(3): 287- 298.

[21] Sweller J. Element interactivity and intrinsic, extraneous, and germane cognitive load. Educational Psychology. 2010; 22(2): 123- 138.

[22] Zhang R, Cook A. Solving complex problems convergent approach to cognitive load. Journal of Educational Technology. 2012; 43(2): 233- 246.

[23] Doyle JK. The cognitive Psychology of systems thinking. Systems Dynamics Review. 1997; 13(3): 253- 265.

[24] Clark R, Nguyen F, Sweller J. Efficiency in learning: Evidence based guidelines to manage cognitive load. San Francisco, CA. Pfeiffer.2006.

[25] Pass F, Van Gog T, Sweller J. Cognitive load theory: New conceptualizations, specifications, and integrated research perspectives. Educational Psychology Review. 2010; 22(2): 115- 121.

[26] Brewster B. Enhancing auditor expertise: Using cognitive load theory to examine a reinforcing feedback loop between knowledge and ability. A dissertation submitted in partial fulfillment of the requirements of the degree of doctor of philosophy in accountancy. University of Illinois, Urban-Champaign. 2008.

[27] Best EE. Building schemata for tax provision learning based on cognitive load theory and constructivism. A dissertation submitted in partial fulfillment of the requirements of the degree of doctor of philosophy in business. Robinson College of Business. Georgia State University. 2013

[28] Pass F, Van-Merrienboer J.The efficiency of instruvtional conditions: An approach to combine mental effort and performance Measures. Human Factors. 1993; 35(4): 737- 743.

[29] De Jong T. Cognitive load theory, educational research, and instructional design: Some food for thought. Journal of Instr Sci. 2010; 38: 105- 134.

[30] Richmond B. Systems thinking: A critical thinking skills for the 1990s and beyond. System Dynamics Review. 1993; 9(2): 934- 947.

[31] Dehdarian A, Mortazavi A, Khandan M, Dorani K, Mahmoudi H, Mashayekhi AN.Developing question sets to assess systems thinking skills.Conference paber, July 2015.pp: 1- 14 , Available from: http://www.researchgate.net/publication/281441003.

[32] Nedra w. The instructional leaders primer in systems thinking. Repruduction supplied by EDRS to the Educational Resources information center(ERIC). Document resume ED 415 203, 1995.pp: 1-20.

[33] Sidney L. Environmental paradigms, biodiversity conservation and critical systems thinking. Systemic Practice and Action Research. 2004; 17(5): 511-515. [34] Gary MS, Wood RE. Mental models, decision making and performance in complex tasks. Conference paber, may 2005.pp: 1- 23 , Available from: http://www.semanticscholar.org.

[35] Richmond B. The thinking in systems thinking : seven essential skills. Waltham, MA: Pegasus communications.2000.

[36] Adam V. Systems Thinking as a major skill of business students: A new teaching concept at the university of Zurich, Switzerland. Journal of Systemic, Cybernetics, and Informatics. 2003; 2(6): 43- 47. 